\title{
Motor Responses to Sudden Limb Displacements in Primates with Specific CNS Lesions and in Human Patients with Motor System Disorders
}

\author{
R. G. LEE AND W. G. TATTON
}

\begin{abstract}
SUMMARY Central feedback pathways for motor control were studied by recording EMG responses to sudden upper limb displacements in humans and monkeys using a precision torque motor to generate step load changes. Normal human subjects showed three shortlatency EMG responses (M1, M2 and M3) which appear to correspond to those recorded from trained monkeys. The $M 2$ and M3 components, thought to represent feedback in supraspinal pathways, were significantly increased when the
\end{abstract}

RÉSUMÉ: Les servo-mécanismes centraux pour le contrôle moteur furent étudiés par enregistrement des réponses EMG aux déplacements soudains du membre supérieur chez l'humain et le singe utilisant un moteur torque de précision pour produire des changements de charge gradués. Les sujets humains normaux montraient trois réponses EMG à courte latence (M1, M2, et $M 3)$ qui paraissaient correspondre à celles enregistrées pour les singes entraînés. Les composantes $M 2$ et $M 3$, qui repré- subjects were instructed to actively compensate for the load changes.

Parkinsonian patients with rigidity showed evidence of markedly increased feedback over the interval for the M2 and $M 3$ responses and appeared to have lost the ability to modulate feedback according to the motor task being performed. The results are discussed with reference to recent research on motor control mechanisms in primates and a tentative model for the basis of Parkinsonian rigidity is proposed.

sentent probablement une rétroaction dans le modèle supraspinal, étaient augmentées significativement quand les sujets étaient avertis de compenser activement pour les changements de charge. Les patients parkinsoniens avec rigidité montraient une augmentation marquée de la rétroaction pendant l'intervalle des réponses $M 2$ et M3 et paraissaient avoir perdu l'abilité à moduler la rétroaction en rapport avec la tâche motrice à être accomplie.
From the Divisions of Medicine and Medical Physiology, Faculty of Medicine, University of Calgary, Alberta, Canada.

Reprint requests to Dr. R. G. Lee, Faculty of Medicine, University of Calgary, Calgary, Alberta, Canada T2N 1N4.

\section{INTRODUCTION}

Much of our understanding of human motor system disorders is based on studies carried out on experimental animals with movement disorders produced by specific central nervous system lesions or chemical agents. Although it has been possible to develop animal models which closely resemble some human diseases such as Parkinsonism (Poirier, 1971; Filion, 1975; Hore et al., 1975), even the most sophisticated models fail to reproduce all the complex disturbances of movement which occur as a result of disease of the human nervous system. The investigator who wishes to fully understand human movement disorders must, therefore, ultimately design studies which can be carried out on human patients.

This leads to a number of immediate problems. While the basic neuroscientist is able to record single unit activity from the depths of the brain, to study the effects of localized lesions, and to confirm his results by precise anatomical studies, the clinical investigator working with human subjects is limited to non-invasive techniques. As a result, there has been some delay in applying new concepts concerning motor control mechanisms to the understanding of human neurologic disorders.

One area in which it has been possible to establish some direct correlations between results in human subjects and experimental animals has been in the elucidation of mechanisms involved in compensating for sudden changes in load or unexpected displacements of an extremity. Early experiments in this 
field were carried out on human subjects over two decades ago by Hammond $(1954,1956)$. He showed that sudden displacements of a handle being grasped by a subject were followed by two separate bursts of electromyographic (EMG) activity during the first 100 milliseconds. The second component was present only when the subject was instructed to actively resist the displacement and was absent during passive displacements.More recently, Marsden et al. (1972, 1973) have shown that human subjects responding to sudden load changes imposed during a flexion movement of the thumb have not only a short latency EMG component commensurate with the monosynaptic spinal reflex, but also show a second component with a latency of 45 to $50 \mathrm{mil}-$ liseconds which they feel indicates the existence of a supraspinal servo-assist mechanism. Responses with similar latencies can be recorded from distal upper limb muscles in man following electrical stimulation of a peripheral nerve (Upton et al., 1971; Milner-Brown et al., 1975). These responses, which have been termed "late reflexes," are potentiated by voluntary effort.

A major advance in understanding motor control mechanisms has resulted from the development of techniques to study the behavior of single neurons from the cerebral cortex and subcortical structures in awake primates trained to perform specific movements. Studies of this type have resulted in the accummulation of a large body of information concerning the role of the motor cortex (Evarts, 1965, 1966, 1968, 1969, 1973), the basal ganglia (Delong, $1971,1972,1973$ ) and the cerebellum (Thach, 1970a, 1970b) in the initiation and control of voluntary movement in non-human primates. The use of torque motors to precisely control the load conditions under which these movements are made has recently added a new dimension to these studies (Evarts, 1973; Atkin et al., 1974; Conrad et al., 1974).

The mechanisms for control of distal upper limb musculature appear to be very similar in man and higher primates. It should therefore be possible to use the results of primate studies as a basis to investigate the manner in which motor control mechanisms are altered in patients with neurologic disorders. In this report we will compare the responses to upper limb displacements produced by sudden load changes in trained monkeys and normal human subjects. Evidence regarding the central pathways mediating one of the components of these responses will be presented. Also, the results obtained in a group of patients with Parkinson's Disease will be described and used to develop a tentative model to explain some of the clinical manifestations of Parkinsonism. Brief reports dealing with some aspects of this work have appeared in previous publications (Tatton and Lee, 1974; Tatton and Lee, 1975).

\section{METHODS}

The technique for recording EMG responses to sudden upper limb displacements in monkeys has been described in detail elsewhere (Evarts, 1973; Evarts and Tanji, 1974; Tatton et al., 1975). The method used to study responses in human subjects was very similar. The subjects grasped a handle which was coupled to a precision torque motor capable of delivering step load changes of 500 grams with a rise time to maximum load of 3.2 milliseconds and a duration of 500 milliseconds.

The monkeys were trained to position the handle in a narrow central zone and received a juice reward for returning the handle through the zone within a specified time following the onset of the displacement. The human subjects were instructed to either relax and allow the handle to be passively displaced (passive task) or to respond by moving the handle as rapidly as possible in a direction opposite to the displacement (active task). The manipulandum was designed to study the responses to displacements at the wrist joint in humans and at either the elbow or wrist in monkeys.

EMG activity was recorded with small surface electrodes placed over the flexor and extensor muscles in the forearm, or for some of the experiments in monkeys, over the

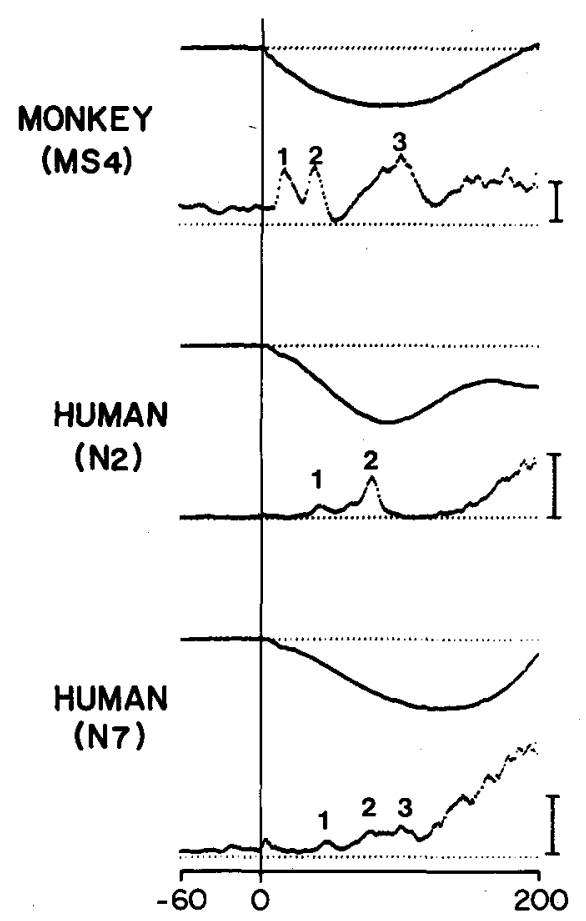

Figure 1-EMG responses to sudden displacements of the elbow in a trained monkey and of the wrist in 2 normal human subjects. Subjects were performing an active movement to oppose the handle displacements. For each pair of tracings, the upper channel presents the average handle position (flexion downward) and lower channel presents the average rectified EMG responses from triceps in monkey and wrist extensors in humans. The vertical line at time 0 indicates time at which torque motor was turned on. The numbers label the M1, M2 and M3 responses (see Text). Calibration bar $=200 \mu \mathrm{V}$.

biceps and triceps. Following full wave rectification, the EMG signals, together with the output of a potentiometer monitoring the handle position were averaged by a PDP 12 computer. For each task responses to 50 or more randomly-timed handle displacements were averaged. A 60 millisecond control interval prior to the step load presentation was also averaged to provide an estimate of the baseline EMG activity. The results reported here were based on studies carried out on four monkeys (Macaca artoides), twelve normal human volunteers, seven patients with Parkinson's Disease and one patient with multiple sclerosis. 

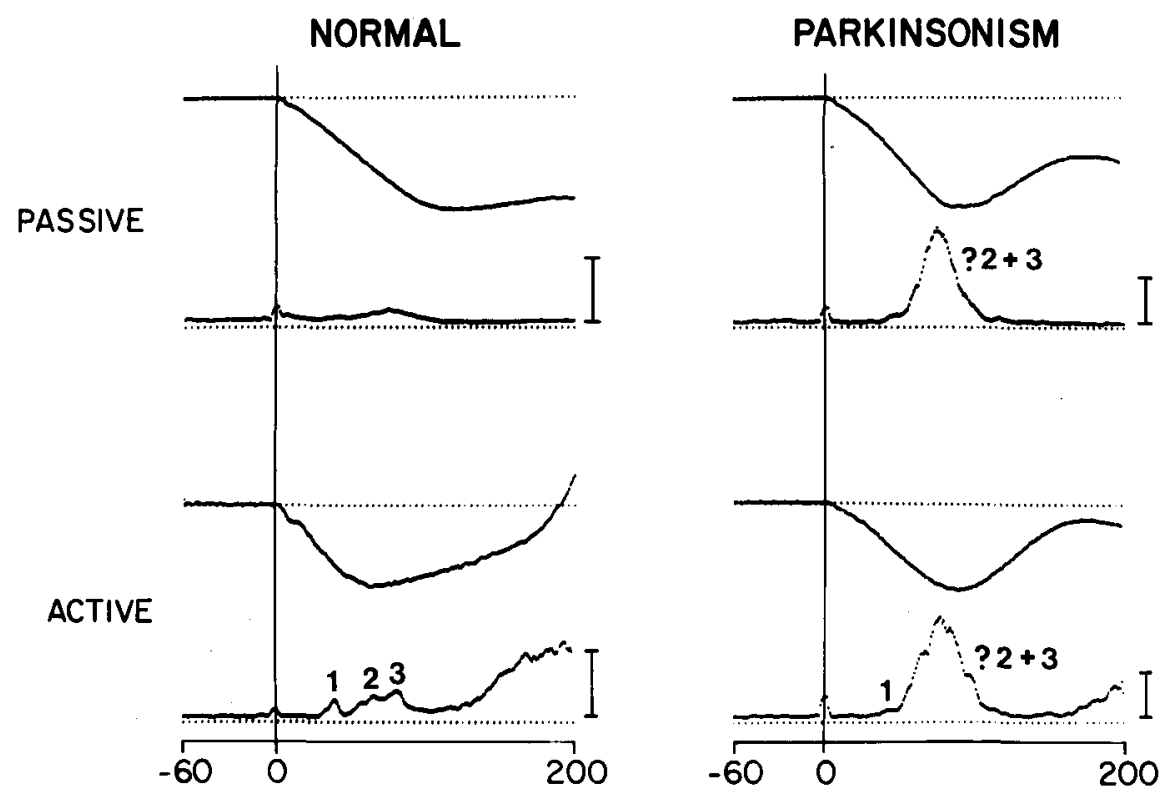

less consistent in humans, but was evident in $40 \%$ of the normal subjects and had a latency of 85 to 95 milliseconds. An M3 component is seen in the recording from subject N7 shown in Figure 1. In some subjects it was continuous with the onset of the later burst of "voluntary" activity beginning 110-160 milliseconds following the onset of handle displacement. If we allow for the longer conduction distances in man, the human M1, M2 and M3 responses appear to be analogous to those recorded from monkeys.

In normal humans there was a definite relationship between the size of the short-latency EMG responses and the volitional "set" of the subject. This is illustrated by responses from another normal human subject (N2) shown in Figure 2. In this example, all three components are increased during the active task as compared to the passive task. As will be shown in a following section, the M1 response usually showed considerably less increase in size than the M2 and M3 responses. These observations in humans, and the fact that the M2 and M3 responses in monkeys were accentuated as they became proficient at performing the task, support the concept that there may be mechanisms for modulating feedback in long-loop reflex pathways to a level which is appropriate for the

ways. Evidence supporting this view will be considered in a subsequent section.

The human subjects showed an M1 response occurring 28 to 32 milliseconds after displacement of the wrist followed by an M2 component beginning at 55 to 60 milliseconds (Figure 1). The M3 response was specific motor task being performed.

Figure 3-Intervals for Determination of the Motor Responses Index. A typical average response to the active task is shown in which the control base line interval, the M1 and the M2-3 intervals are shaded and identified. The motor response index was calculated according to:

MRI $\frac{\mu_{R}-\mu_{C}}{\mu_{C}}$, where

$\mu_{\mathrm{R}}=$ mean voltage over defined response interval in microvolts/second

$\mu_{\mathrm{C}}=$ mean voltage over the control interval in microvolts $/$ millisecond

tivity with a latency of 70 to 100 milliseconds which appeared to represent the voluntary response associated with return of the handle. The first component (M1) is appropriately timed for the monosynaptic spinal reflex and is probably analogous to the tendon jerk (Evarts and Tanji, 1974). It has been proposed that the M2 and $\mathrm{M} 3$ responses are mediated by long-loop reflexes utilizing supraspinal feedback path-

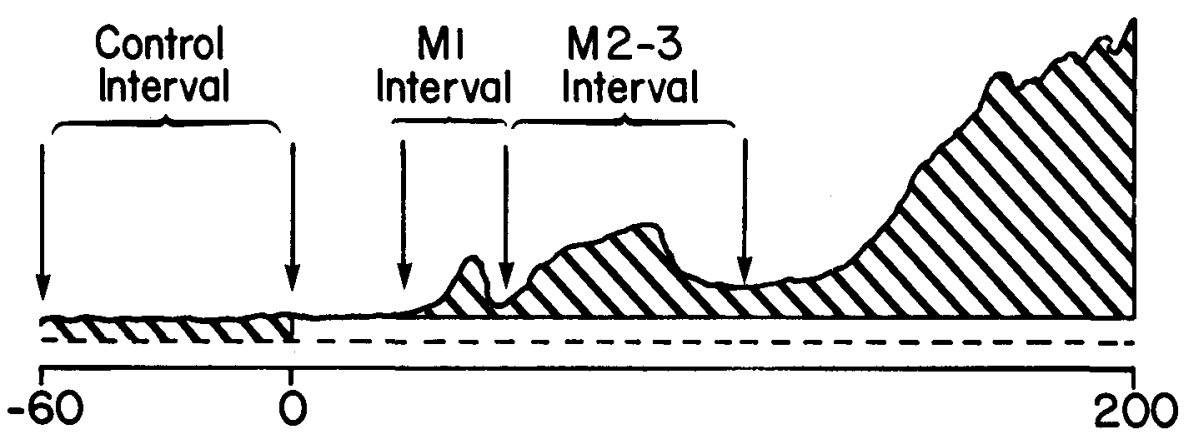




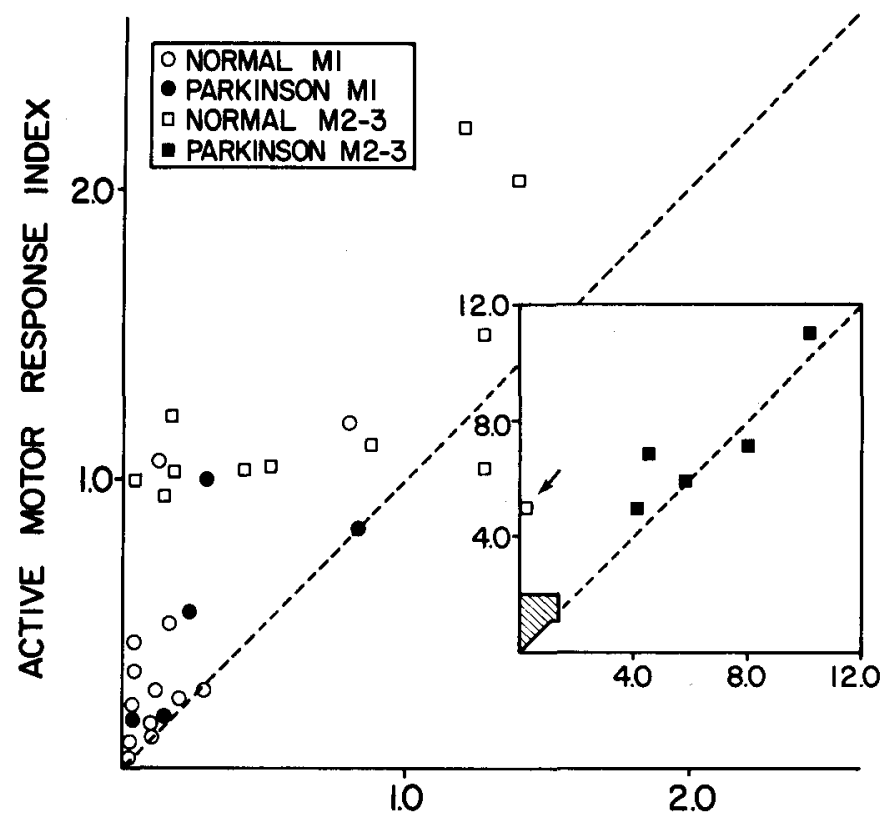

PASSIVE MOTOR RESPONSE INDEX

Figure 4-Motor Response Index in Active Versus Passive Tasks. Each symbol represents the relative MRI for a given response interval for the active (ordinate) versus passive task (abscissa) determined for a single subject. Points falling above the dashed diagonal indicate increased responses for the active task in comparison to the passive task. The inset graph has a lengthened scale to accommodate the M2-3 responses in rigid Parkinsonian patients. The shaded area on the inset graph defines the extent of the symbols plotted on the main graph. The arrow locates one normal whose values fell outside the shaded area.

\section{TRAINED MONKEY - POST CENTRAL LESION}

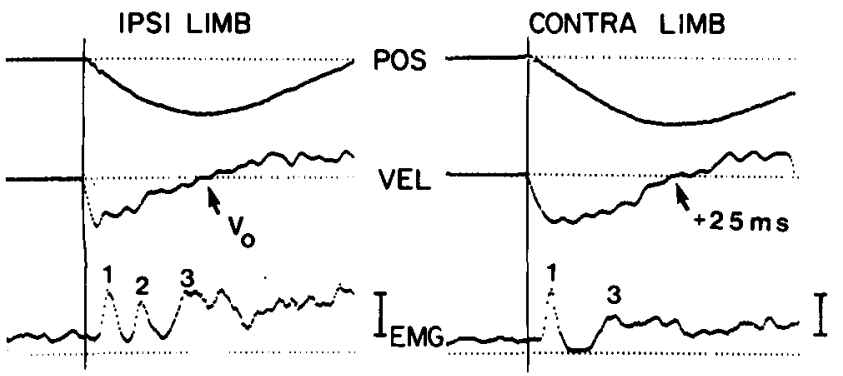

\section{M.S. PATIENT - UNILATERAL DORSAL COLUMN} LESION

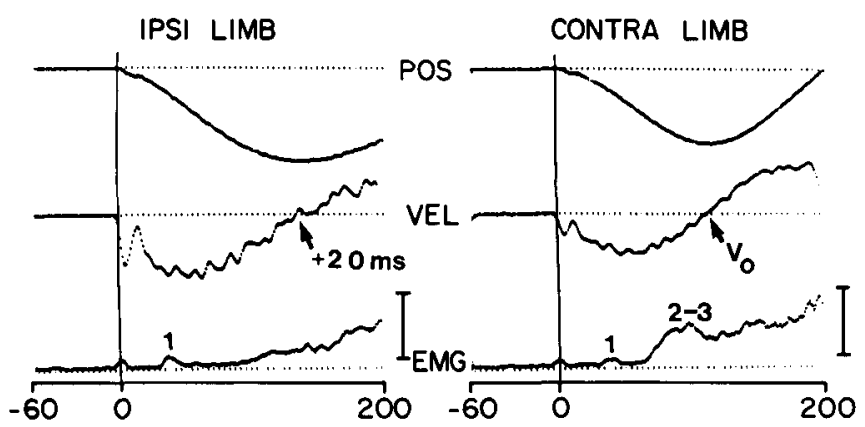

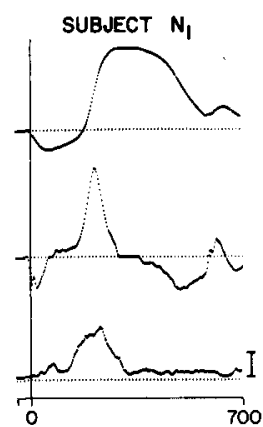

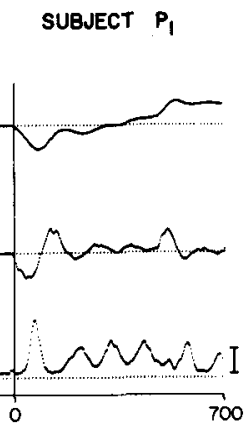

SUBJECT $P_{B}$

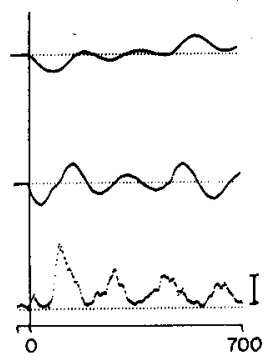

Figure 5-Average handle position (top trace), average velocity of handle movement (middle trace) and average rectified EMG activity (lower trace) from wrist extensors for 700 mil liseconds following flexor displacements of the handle for two Parkinsonian patients (P1 and P5), and a normal subject (N1). Subjects were actively responding to handle displacements with extensor movement of wrist. Each trace is an average of 50 responses. Calibration bar $=200 \mu \mathrm{V}$. Note the rhythmical oscillations in EMG activity following the increased responses in the M2-3 interval for the Parkinsonian subjects.

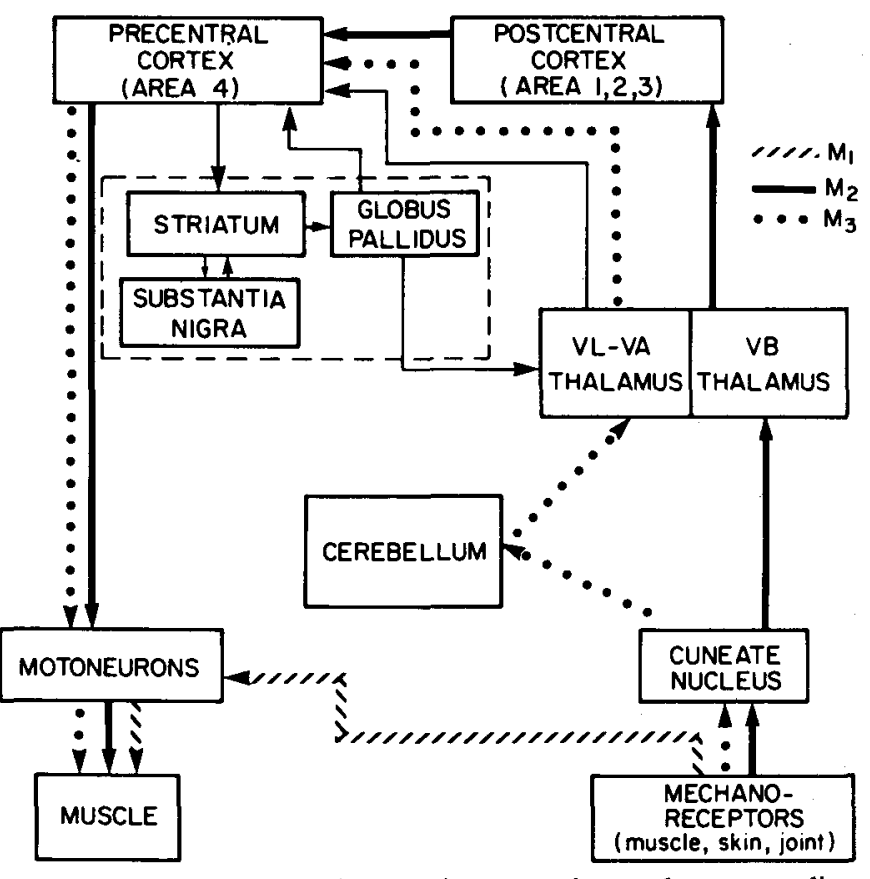

Figure 7-Block schematic showing tentative pathways mediating the M1, M2 and $\mathrm{M} 3$ responses and their relationship to the basal ganglia which are enclosed within the interrupted line.

Figure 6-EMG responses to sudden upper limb displacements in a monkey with a unilateral lesion of the postcentral cortex and in a multiple sclerosis patient with clinical signs indicating a unilateral dorsal column lesion. Each set of traces represents an average of 50 responses. EMG activity from triceps in the monkey and the wrist extensors in the human subject. Torque motor turned on at time 0 . Calibration bar $=200 \mu \mathrm{V}$. The upper trace is average handle position, the middle trace is the average handle velocity and the lower trace is the rectified EMG activity. $V_{0}$ indicates the time of zero handle velocity which occurs later in the affected limb of both the lesioned monkey and the multiple sclerosis patient. 
Responses in Parkinsonian Patients

In the Parkinsonian patients, the M1 responses were similar in amplitude and latency to those seen in normal subjects, a finding in accord with clinical observations that tendon reflexes are usually not altered in Parkinsonism. However, a marked difference from normal was seen in the M2 and M3 segments of the response in patients manifesting cogwheel rigidity. Figure 2 shows responses from one of the Parkinsonian patients. Beginning 60 milliseconds following displacement of the handle and lasting for approximately $\mathbf{4 0}$ milliseconds, there was a very large, long duration EMG response with an amplitude three or four times greater than either the M2 or M3 responses in normal subjects. Furthermore, this component was very large during passive displacement of the wrist and there was little further increase in size during performance of the active task. This patient was a 56-year-old woman with relatively mild Parkinsonism manifested by slight cogwheel rigidity and slowness of movement in the distal parts of the right upper extremity. She did not have any clinically detectable tremor. The responses shown in Figure 2 were recorded from the extensor muscles in the right forearm.

Responses similar to these were recorded from all five Parkinsonian patients in whom the clinical findings included some degree of rigidity in one or both upper extremities. Some of these patients exhibited varying degrees of rest tremor and generalized bradykinesia as well. In contrast, we have been able to study two patients who have Parkinsonian rest tremor without clinical changes in muscle tone. Neither of these patients showed significant accentuation of their EMG responses.

The large second component of the averaged EMG responses in the Parkinsonian patients had its onset at the same time as M2 and spanned the time period which includes the M2 and M3 responses in normal subjects. Therefore, for quantitative analysis, the interval including these abnormally large responses in Par-

TABLE 1

MOTOR RESPONSE INDICES (Mean + S.D.) for the MI and M2-3 components during passive and active tasks in normal subjects and in Parkinsonian patients with rigidity.

\begin{tabular}{lcccc} 
& \multicolumn{2}{c}{$\frac{\mathrm{M1}}{\text { passive }}$} & $\frac{\text { active }}{\text { passive }}$ & $\frac{\mathrm{M2-3}}{\text { active }}$ \\
$\frac{\text { Normal Subjects }}{(\mathrm{N}=12)}$ & $0.22 \pm 0.16$ & $0.30 \pm 0.21$ & $0.72 \pm 0.50$ & $1.68 \pm 0.74$ \\
$\frac{\text { Rigid Parkinsonians }}{(\mathrm{N}=5)}$ & $0.34 \pm 0.21$ & $0.62 \pm 0.30$ & $6.47 \pm 2.14$ & $7.30 \pm 2.39$
\end{tabular}

kinsonian patients and the M2 and M3 responses in normals will be termed the M2-3 interval.

To compare the responses from the Parkinsonian patients with those of normal subjects, we calculated the motor responses index (MRI) which related the total EMG activity, or motoneuron excitability, during the responses to the control EMG activity during the 60 millisecond interval prior to the onset of the displacement. On the basis of measurements of latencies of responses in normal subjects, the M1 interval was defined as that part of the response occurring between 28 and 58 milliseconds and the M2-3 interval was taken as the segment between 58 and 108 milliseconds (Figure 3). Using the mean voltage during the control interval as a baseline, we calculated the area of the response extending above this baseline during the M1 and M2-3 intervals. The MRI was expressed as a ratio of the mean voltage above baseline (microvolts/millisecond) during the defined response intervals to the mean voltage during the control interval.

The average results for the 12 normal subjects and five Parkinsonian patients are presented in Table 1. During the passive task the MRI for the M2-3 interval in the patients was approximately nine times larger than the corresponding value for normal subjects. In the control group there was significant accentuation of the M2-3 component during the active task, the MRI increasing to a value more than twice what it was with passive displacement. In the Parkinsonian patients, the active task resulted in only a slight increase in the size of the M2-3 component.
The M1 component was slightly larger in Parkinsonian patients than in normal subjects and in both groups there was some increase in size from the passive to the active task.

The degree of passice-actice modulation is shown in Figure 4 where the motor response indices for each subject have been plotted with the value during the passive task on the abscissa and the index during the active task on the ordinate. In this display, if there is no change between the passive and active conditions, the points should fall on or near the $45^{\circ}$ diagonal. This is the case for most of the M1 components for both normal and Parkinsonian patients. In contrast, the points for the normal M2-3 responses are located well above the diagonal indicating significant modulation from the passive to the active task. The M2-3 components for the Parkinsonian patients were so large that a different scale had to be used to plot the motor response indices, and the points representing these are shown on the inset plot in Figure 4. All of these points are close to the $45^{\circ}$ diagonal showing that this group failed to modulate the increased responses to any significant degree.

We do not yet have sufficient data to draw firm conclusions concerning the effect of anti-Parkinson therapy on these abnormally large responses during the M2-3 interval. However, we have had an opportunity to study one controlled patient before, and following, treatment with L-Dopa. Before treatment the MRI values for the M2-3 interval were 6.07 with passive displacement and 5.82 during the active task. Following six weeks of treatment with L-Dopa 
(maximum dose of 4.0 grams/day) the clinical rigidity was moderately reduced and the MRI values for the M2-3 interval were 3.05 (passive) and 3.91 (active). These results, although preliminary, provide further evidence for a correlation between clinical rigidity in Parkinsonism and the increased feedback during the M2-3 interval.

So far we have restricted our attention to events occurring within the first 110 milliseconds following sudden load changes. However, inspection of the voluntary EMG activity occurring beyond 110 milliseconds during the active task reveals further abnormalities in the Parkinsonian patients. In the average EMG response from subject $P 1$, shown in Figure 5, there is a rhythmical oscillation of approximately 10 $\mathrm{Hz}$ which is initiated by the large M2-3 response. This is not sufficient to cause a clear oscillation in the position record but the oscillation is clearly evident in the velocity of handle movement, shown on the middle channel, which correspond to the rhythmical peaks of EMG activity. This particular patient did not have any tremor on clinical examination.

Also shown in Figure 5 are results from another Parkinsonian patient (P5) who had a prominent $5 \mathrm{~Hz}$ rest tremor as well as mild rigidity and bradykinesia. The EMG trace shows a rhythmical $5 \mathrm{~Hz}$ oscillation in the voluntary activity with a corresponding oscillation in the handle velocity. Since the handle displacements were imposed at random times, it is unlikely that the spontaneous rest tremor would appear in the averaged responses unless its timing was being modified by the proprioceptive input associated with the sudden load changes. The normal subjects did not evidence an oscillation in their velocity or EMG traces as is illustrated by the records for subject N1 in Figure 5. These results suggest that, in the presence of an overcompensated feedback system, the peripheral mechanoreceptor input may either reset an existing $5 \mathrm{~Hz}$ rest tremor, or initiate a $10 \mathrm{~Hz}$ oscillation which is not present in the resting state. This finding is in accord with earlier evidence that in some Parkinsonian patients tremor frequency may double from $5 \mathrm{~Hz}$ to $10 \mathrm{~Hz}$ in response to either sensory input or during volitional movements (Landau, 1974).

\section{Anatomical Basis and Functional Significance of the $M 2$ and $M 3$ Responses}

The M1 response in monkeys (Tanji and Evarts, 1974) and humans is appropriately-timed to be mediated by the monosynaptic spinal reflex. Phillips (1969) proposed that during load compensation the spinal reflexes could be reinforced by a transcortical feedback loop. On the basis of the sequential minimum latencies of single neurons in the postcentral and precentral cortex to upper limb displacements, Evarts (1973) suggested that the second component of the EMG response (M2 in our recordings) is mediated by a transcortical pathway. These observations have been confirmed by other investigators (Tatton and Gerstein, unpublished observations). Furthermore, a recent study by Tatton et al. (1975) has shown a selective loss of the M2 response in monkeys with lesions of the postcentral cortex. Recordings from a monkey with a postcentral cortical lesion are shown in Figure 6. Normal M1, M2 and M3 responses are seen for the limb on the same side as the lesion. In the contralateral limb, M1 and $\mathrm{M} 3$ appear normal, but the $\mathrm{M} 2$ is missing. The lesioned monkey showed surprisingly little motor deficit, but the response to handle displacement was slightly slower in the affected limb and examination of the velocity indicating the time at which the direction of handle movement was reversed, occurred 25 milliseconds later than in the ipsilateral limb.

The short latency of the M2 response suggests that it utilizes a fast conducting afferent pathway such as the dorsal column and medial lemniscus. To investigate the role played by the dorsal columns, we recorded EMG responses from a young woman with multiple sclerosis, and clinical signs suggesting a unilateral lesion in the fas- ciculus cuneatus. The results are shown in the lower part of Figure 6. In the clintically normal arm, contralateral to the lesion, there is a second peak of EMG activity at a time appropriate for the M2 and M3 responses. In the ipsilateral arm, there is an M1 but no evidence of either an M2 or M3. As in the lesioned monkey, the absence of M2 and $\mathrm{M} 3$ responses is associated with a significant delay in returning the handle.

The origin of the M3 component is less clearly established in primates, but there is evidence in cats for a second feedback loop to motor cortex via the cerebellum. Murphy et al. (1974) have shown changes in activity of motor cortical neurons occurring at two latencies following muscle stretch (11 milliseconds and 17 milliseconds). The later response was reversibly blocked by cooling the interpositus nucleus of the cerebellum.

Our results provide further evidence for the existence of "longloop" reflexes or feedback loops which may play a role in the control of voluntary movement in monkeys and man. The functional significance of these reflexes is not yet fully established, but evidence to date suggests that they represent one of the mechanisms by which motoneuron activity can be adjusted to compensate for unexpected load changes occurring during the course of volitional movement. Some of the major components of the motor system in man and primates and the pathways proposed for these short-latency EMG responses are shown in Figure 7. Three proposed routes by which feedback from sensory receptors in a limb may influence motoneurons controlling muscles in that limb are shown. The first of these is the monosynaptic reflex pathway which is most likely responsible for the M1 response in our recordings. The other two pathways convey information back to motor cortex and then to motoneurons via descending motor pathways, particularly the corticospinal tract in the case of distal upper limb muscles (Kuypers, 1964). The ascending pathway for the M2 response, shown as a solid 
line in Figure 7, is believed to include the dorsal columns, medial lemniscus, ventrobasal thalamus, and postcentral cortex. Supporting evidence for this has been provided by the selective loss of the M2 component in monkeys with postcentral lesions and in a human subject with a unilateral dorsal column lesion. The proposed pathway for the M3 component includes the dorsal column, interpositus nucleus of cerebellum, superior cerebellar peduncle, and ventrolateral thalamus (VL-VA).

\section{A Tentative Model for \\ Parkinsonian Rigidity}

How can we relate our observations of an accentuated response during the M2-3 interval in Parkinsonian patients to what is already known concerning the pathophysiology of the disease? Included within the dashed line in Figure 7 are the major structures collectively referred to as the basal ganglia and some of their known connections to other components of the motor system. The substantia nigra projects to the striatum, which in turn sends projections to the globus pallidus. One of the major outflows from the globus pallidus goes to the VL-VA complex of the thalamus from which fibres project to layers 2 and 3 of the precentral motor cortex (Nauta and Mehler, 1969; Kemp and Powell, 1971). The precentral cortex may also receive some direct input from the globus pallidus (Kierit and Kuypers, 1975) and, together with other cortical areas, sends projections back to the striatum.

It is known that some of the clinical manifestations of Parkinsonism are associated with structural and chemical abnormalities in the basal ganglia. Pathologically, degeneration and loss of neurons in the substantia nigra is a constant feature, and similar changes are frequently seen in the striatum and globus pallidus (Greenfield, 1963). Hornykiewicz and his coworkers have (1960) demonstrated a deficiency of striatal dopamine in the brains of Parkinsonian patients. Furthermore, it has been shown in monkeys that lesions in the ventral medial mesencephalic tegmentum which in- clude parts of the nigral-striatal pathway (Poirier, 1971) or reversible cooling of the globus pallidus (Hore et al., 1975) can produce Parkinsonian-like motor deficits.

The increased feedback during the M2-3 interval does not necessarily indicate increased transmission in the M2 and M3 pathways. Conceivably, rigid Parkinsonian patients could have transmission in a further long-loop pathway which is not operative in normal individuals. Yet, the exact coincidence of its time course with that of the normal longloop reflexes supports the interpretation of increased transmission in the M2 and M3 pathways. Further, we have examined response planes constructed for sequences of individual displacements and have found that the increased average response has separable components which correspond in time to the normal M2 and $\mathrm{M} 3$ responses.

Accepting that transmission is increased in the M2 and M3 pathways and that both these pathways involve the motor cortex, a point for possible interaction between the basal ganglia output and the proposed pathways for the M2 and M3 reflexes is in the superficial layers of the motor cortex. Primate motor cortical neurons are known to receive inputs from peripheral mechano-receptors (Rosen and Asanuma, 1972; Wiesendanger, 1973) which appear to be topographically organized in manner appropriate to a highly focused feedback system. Further, Evarts and Tanji (1974) have shown that the activity of motor cortical neurons in monkeys is modulated in relation to the prior instruction given to the animals while they are performing tasks similar to those of the present study. The fact that the M2-M3 responses increase in normal human subjects during performance of an active task is also in accord with selective modulation of mechanoreceptor inputs to the motor cortex. Therefore we propose a tentative model in which basal ganglia input to the motor cortex modulates mechanoreceptor information arriving from the periphery so as to adjust the gain in the feed- back system to a level appropriate for the motor task being performed. Recent studies of the activity of basal ganglia neurons in awake monkeys performing specific upper limb movements (Delong, 1971, 1972, 1973) have shown that activity in these structures occurs prior to voluntary movements but have not elucidated the manner in which basal ganglia input influences motor cortical activity. Yet it is known that globus pallidus output is mainly facilitatory to motor cortical neurons in the cat and can modulate their responses to other inputs (Newton and Price, 1975). Damage within the basal ganglia which resulted in increased activity of globus pallidus neurons could create a situation in which the gain in transcortical feedback loops would be set constantly at maximum levels. Inputs from the periphery would then result in an exaggerated response which could account for the accentuation of the M2-3 components and for Parkinsonian rigidity. Support for this model is provided by Filion et al.'s (1975) finding that a lesioned monkey with prominent rigidity showed abnormally high rates of neuronal discharge in the globus pallidus. Further, the rigidity and the high rates of globus pallidus discharge were both reversed by apomorphine.

Further studies are required to test this model, to relate changes in EMG responses to other clinical manifestations of Parkinsonism, and to determine further what effect treatment with anti-Parkinson drugs has on the reflex abnormalities. However, our results to date suggest that this approach is one which can be readily applied to human subjects and which, with further development, may provide a useful technique for assessment of patients with motor system disorders. It also provides the opportunity to establish direct correlations between results obtained from animal experiments and studies on human subjects.

\section{DISCUSSION}

Upton (McMaster) reported related findings by McComas and himself, namely that the V-2 response to supramaximal mixed nerve stimulation to a muscle during effort of $\mathbf{3 0}$ Newcastle Parkinsonians were particularly 
large, did not habituate and were reduced by L-dopa.

McComas (McMaster) stressed the possibility of input from skin receptors to the M1, $M 2$ and M3 responses, since Sica, himself and Upton had been able to superimpose complex waves onto voluntary EMG's by making very small skin displacements. Tatton replied that he and Lee had also noticed influence from skin in humans and primates that had to grasp a handle, which provided strong cutaneous and mechanoreceptor input on the flexor, but not on the extensor surfaces. When the mediam and ulnar nerves at the wrist were blocked, leaving muscle receptors intact, human subjects could still report on wrist position after palmar cutaneous sensibility had been lost. This procedure surprisingly increased long-loop feedback in flexor muscles, which suggests that cutaneous inputs can inhibit feedback from related muscles, an arrangement that could stabilize the load compensating system.

Phillips asked Tatton and Lee to specify what alternative interpretations they may have had in mind to a transcortical loop for generation of the $\mathrm{M} 2$ response, as suggested in the presentation. Tatton suggested that removal of postcentral cortex could have abolished M2 by disfacilitation of known spinal or brainstem pathways.

Hore contrasted Tatton's result with that obtained in recent experiments with Vilis in Brooks' laboratory in which M1, M2 or M3 responses to arm perturbations of two monkeys were retained after cooling the globus pallidus.

Tatton suggested that this finding could perhaps be coupled to the observed loss of muscle reciprocity. He suggested that the output of the basal ganglia might work like a switchboard, where feedback can affect command systems to choose between appropriate compensating mechanisms, as in invertebrate systems. Girvin asked for interpretation of the increased M1 wave when M3 decreased after postcentral lesions. Tatton replied the labile time course of M3 may relate to $\mathrm{M} 3$ being regarded as the beginning of "voluntary" activity. Its latency decreased slightly after postcentral lesions, when the monkey was trying to make a faster return to get his reward. This was true even when the animals had been rewarded after continually harder criteria over 10 months after the lesions, offsetting the fear that M2 loss might have been "motivational".

\section{General Discussion on Transcortical Re-} flexes

Stein: (Edmonton) I have a couple of comments which relate to, and elaborate, on what John Murphy has just talked about, and which also return to some of the general considerations we were discussing this morning. I have attempted to include the detailed properties of muscles and of muscle spindles in order to determine the overall properties of the feedback pathways. Parveen Bawa, Allan Mannard and I measured the dynamic characteristics of muscle, which is one of the boxes in John Murphy's diagram, while Peter Matthews and I studied muscle spindles

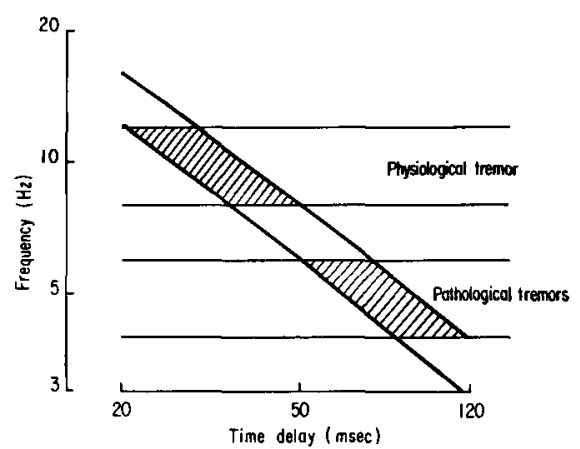

Figure-Computed frequencies of oscillation for muscle reflex pathways involving a fast muscle (upper curve) or a slow muscle (lower cruve) as a function of the delay in the reflex pathway. The pairs of horizontal lines indicate the usual limits of physiological tremors $(8-12 \mathrm{~Hz}$ ) and of pathological tremors (4-6 Hz) such as in Parkinson's Disease or cerebellar disorders. Details of the model will appear in J. Math. Biol. and the results shown here have also been submitted to J. Physiol.

which represent another box. Finally, we have considered feedback pathways of varying lengths and hence having varying time delays. If the time delay in the reflex pathway is varied over the range that we have been considering, the frequency of tremor that would result from this feedback pathway under various assumptions can be calculated. The upper curved line in the figure shows results computed for quite a fast muscle, such as plantaris in the hind limb. The lower line shows results for quite a slow muscle such as soleus in the hind limb. Note that quite wide variation in muscle properties (about 5-fold) does not change the frequency too greatly. If there is a short latency pathway, such as in the spinal monosynaptic reflex (i.e., under 50 msec.) the frequency of tremor from the feedback pathway will be within the normal range of physiological tremor $(8-12 \mathrm{~Hz})$ which is indicated by the horizontal lines in this figure. However, if you have a longer latency pathway (over $50 \mathrm{msec}$.) involving higher centres (cortex and/or cerebellum), again under quite wide variation in muscle properties, the tremor is just in the range that we were discussing earlier $(4-6 \mathrm{~Hz})$ which is characteristic of Parkinsonian tremor and cerebellar disease.

These considerations on the reflex pathways lead me to speculate on the following idea concerning these two major types of reflex pathways which tend to oscillate at $4-6 \mathrm{~Hz}$ in the case of long pathways, or 8-12 Hz in the case of the short pathways. Perhaps, the brain has evolved in such a way as to damp out, or control these tremors, and because we have these two major types of tremors possible, two centers have evolved to eliminate these tremors. However, when some pathological process affects these centers, the centers no longer generate appropriate signals and become not tremor controlling centers but tremor generating centers, as Yves Lamarre was discussing this morning.
Hore emphasized once more that M2 responses could not be exclusively due to transcortical reflexes. Brooks agreed that it was not exclusive, but that there was strong presumptive evidence that the demonstrated responses in precentral cortex participated in the generation of the $M 2$ response. However, the experiment of excising motor cortex still had to be done.

Murphy reiterated the importance of multiple feedback loops and pointed out that no matter whether they are running through spinal motoneurons or through the motor cortex, their activation by peripheral feedback depends on what Jasper called "state".

Jasper pointed out that we still do not know how the joint afferents fit into the position feedback system, as opposed to the force or velocity feedback systems that may be operated by the muscle sense organs.

Beck (Edmonton) recounted Rank's description of hippocampal unit discharges during "appetitive" behaviour, or "consummatory" behaviour, whereas others responded selectively to unexpected contingencies. He wondered whether these match-mismatch types of hippocampal responses might be similar to sensorimotor cortex during feed-back in transcortical servo loops.

\section{ACKNOWLEDGMENTS}

The authors wish to thank G. Mildenberger, $D$. Mauser and D. White for technical assistance. This work was supported by Medical Research Council of Canada grants MA 4209 (R. G. Lee) and MA 5218 (W. G. Tatton). W. G. Tatton is a Medical Research Council of Canada Scholar.

\section{REFERENCES}

ATKIN, A., BROOKS, V. B. and PISCHINGER, S. (1974). A torque device for studies of primate limb movements. Physiology and Behaviour 12, 127-129.

CONRAD, B., MATSUNAMI, K., MEYERLOHMANN, J., WIESENDANGER, $M$. and BROOKS, V. B. (1973). Cortical load compensation during voluntary elbow movements. Brain Res. 71, 507-514.

DELONG, M. (1971). Activity of pallidal neurons during movement. J. Neurophysiol. 34, 414-427.

DELONG, M. (1972). Activity of basal ganglia neurons during movement. Brain Res. 30, 127-135.

DELONG, M. (1973). Putamen: Activity of single units during slow and rapid arm movements. Science 179, 1240-1242.

EHRINGER, H. and HORNYKIEWICZ, O. (1960). Verteilung von Norandrenalin und Dopamin (3-Hydroxytyramin) im Gehirn des Menschen und ihr Verhalten bei Erkrankungen des extrapyramidalen Systems. Klin. Wschr. 38, 1236.

EVARTS, E. V. (195). Relation of discharge frequency to conduction velocity in pyramidal tract neurons. J. Neurophysiol. 28, 216-228.

EVARTS, E. V. (1966). Pyramidal tract associated with a conditioned hand movement in the monkey. J. Neurophysiol. 29, 1011-1027.

EVARTS, E. V. (1968). Relation of pyramidal tract activity to force exerted during voluntary movement. J. Neurophysiol. 31, 14-27.

EVARTS, E. V. (1969). Activity of pyramidal tract neurons during postural fixation. J. Neurophysiol. 32, 375-385. 
EVARTS, E. V. (1972). Contrasts between activity of precentral and postcentral neurons of cerebral cortex during movement in the monkey. Brain Res. 40, 25-31.

EVARTS, E. V. (1973a). Motor cortex reflexes associated with learned movement. Science 179 , 501-503.

EVARTS, E. V. and TANJI, J. (1974). Gating of motor cortex reflexes by prior instruction. Brain Res. 71, 479-494.

FILION, L., LAROCHELLE, L. and POIRIER, L. J. (1974). Ghobus pallidus unit activity in a monkey with a Parkinson-like syndrome. Effect of apomorphine. Clin. Res. 22, 5, 754a.

GREENFIELDS. Neuropathology, E. Blackwood, E. (Edward Arnold, London, 1963), pp. 582-585.

HAMMOND, P. H. (1954). Involuntary activity in biceps following the sudden application of velocity to the adbucted forearm. J. Physiol. 127, 17-18P.

HAMMOND, P. H. (1956). The influence of prior instruction to the subject on an apparently involuntary neuro-muscular response. J. Physiol. (Lond.) 132, 17-18P.

HORE, J., MEYER-LOHMANN, J. and BROOKS, V. B. (1975). Effects of cooling globus pallidus on monkey arm movements. Can. Physiol. 6, 30.

KEMP, J. M. and POWELL, T. P. S. (1971). The connections of the striatum and globus pallidus; synthesis and speculation. Phil. Trans. B. 262, 441-457.

KIERIT, J. and KUYPERS, H. J. M. (1975). Basal forebrain and hypothalmic connections to frontal and parietal cortex in the Rhesus monkey. Science $187,660-662$

KUYPERS, H. G. J. M. (1964). The descending pathways to the spinal cord, their anatomy and function. In J. C. Eccles and J. P. Schade (eds.) Organization of the Spinal Cord, Progress in Brain Research, Vol. 11, Elsevier, Amsterdam, 1964, pp. 178-202.

LANDAU, W. M. In Neurological Pathophysiology, S. G. Eliasson, A. L. Prensky and W. B Hardin, Jr. (eds.) Oxford University Press, 1974, p. 132 .

MARSDEN, C. D., MERTON, P. A. and MORTON, H. B. (1972). Servo action in human voluntary movement. Nature 238, 140-143.

MARSDEN, C. D., MERTON, P. A. and MORTON, H. B. (1973). Latency measurements compatible with a cortical pathway for the stretch reflex in man. J. Physiol. 230, 58-59P.

MILNER-BROWN, H. S., STEIN, R. B. and LEE, R. G. (1975). Synchronization of human motor units: possible roles of exercise and supraspinal reflexes. Electroenceph, and Clin. Neurophysiol. $38,245-254$.

MURPHY, J. T., WONG, Y. C. and KWAN, H. C. (1974). Distributed feedback systems for muscle control. Brain Res. 71, 495-505.

NAUTA, W. J. H. and MEHLER, W. R. (1969). Fiber connections of the basal ganglia. In $G$. Crane and R. Gardner, Jr. (eds.), Psychotrophic Drugs and Dysfunction of the Basal Ganglia, Public Health Services Publication No. 1938 U.S. Government Printing Office, Washington, D.C., pp. 68-74.

NEWTON, R. A. and PRICE, D. D. (1975). Modulation of cortical and pyramidal tract induced motor responses by electrical stimulation of the basal ganglia. Brain Res. 85, 403-422.

PHILLIPS, C. G. (1969). Motor apparatus of the baboon's hand. Proc. Roy. Soc. B. 173, 183-198.
POIRIER, L. J. (1971). The development of animal model for studies in Parkinson's disease. In " $\mathbf{R e}$ cent Advances in Parkinson's Disease" edited by F. H. McDowell and C. H. Markham, Blackwell, Oxford, pp. 83-117.

ROSEN, I. and ASANUMA, H. (1972). Peripheral afferent inputs to the forelimb area of monkey motor cortex: input-output relations. Exp. Brain Res. 14, 257-273

TATTON, W. G. and LEE, R. G. (1974). Motor responses to sudden displacements of the wrist in normal humans and Parkinsonian patients. Clin. Res. 22, 5, 755a.

TATTON, W. G. and LEE, R. G. (1975). Motor responses to sudden upper limb displacements in primates, humans and Parkinsonian patients. Canada Physiology 6, 1, 60.

TATTON, W. G., FORNER, S. D., GERSTEIN, G. L., CHAMBERS, W. W. and LIU, C. N. (1975). The effect of postcentral cortical lesions on motor responses to sudden upper limb displacements in monkeys. Brain Res. (In Press).

THACH, W. T. (1970a). Discharge of cerebellar neurons related to two maintained postures and two prompt movements. I. Nuclear cell output. J. Neurophysiol. 33, 527-536.

THACH, W. T. (1970b). Discharge of cerebellar neurons related to two maintained postures and two prompt movements. II. Purkinje cell output and input. J. Neurophysiol. 33, 537-547.

UPTON, A. R. M., McCOMAS, A. J. and SICA, R. E. P. (1971). Potentiation of "late" responses evoked in muscles during effort. J. Neurol. Neurosurg. Psychiat. 34, 699-711.

WIESENDANGER, M. (1973). Input from muscle and cutaneous nerves of the hand and forearm to neurones of the precentral gyrus of baboons and monkey. J. Physiol. (Lond.) 228, 203-219. 